\title{
Medical approach to the treatment of feline injection site sarcoma with masitinib: a case report
}

\author{
Jean-Marie Ledoux' \\ Pascal Brun ${ }^{2}$ \\ Tom Chapuis ${ }^{2}$ \\ Paul Dumas ${ }^{3}$ \\ Jean Guillotin ${ }^{4}$ \\ 'Veterinary Surgery, Lys-Lez-Lannoy, \\ ${ }^{2} A B$ Science, Paris, ${ }^{3}$ Laboratoire \\ de Pathologie Vétérinaire du \\ Nord, Annœullin, ${ }^{4}$ Laboratoire \\ Départemental Public, Villeneuve \\ d'Ascq, France
}

This article was published in the following Dove Press journal:

Veterinary Medicine: Research and Reports

5 September 2014

Number of times this article has been viewed
Correspondence: Jean-Marie Ledoux Veterinary Surgery, 17 rue Jules Guesde, 59390 Lys-Lez-Lannoy, France Fax +33320759185

Email ledoux.jean-marie@wanadoo.fr

\begin{abstract}
Feline injection site sarcoma is a common tumor among cats, for which existing medical treatments do not prove to be entirely satisfactory. In this tumor, the platelet-derived growth factor receptor, a tyrosine kinase receptor, is frequently hyperactivated. In the past, clinical case reports with imatinib, a tyrosine kinase inhibitor (TKI), have demonstrated tumoral stabilization. Here we describe the use of another TKI, masitinib, which specifically inhibits c-Kit, platelet-derived growth factor receptor, and Lyn, and is currently licensed for veterinary use in canine mast cell tumors. The therapeutic results were initially satisfactory, with regression of the tumor followed by tumoral recurrence which was stabilized and moderately reduced. Further studies are suggested, in order to evaluate the relevance of TKIs in the treatment and prevention of recurrences of feline injection site sarcoma. Tumoral stabilization by means of an inexpensive and reasonably well tolerated treatment would prove to be of true therapeutic relevance, in particular for inoperable feline injection site sarcomas. Another indication for such TKIs could be in preoperative treatment as a means of facilitating surgical excision by reduction of adhesions.
\end{abstract}

Keywords: fibrosarcoma, imatinib, platelet-derived growth factor receptor, tyrosine kinase receptor

\section{Introduction}

Feline injection site sarcoma (FISS) is a common subcutaneous tumor, and occurs among subjects aged on average between 8 and 10 years. ${ }^{1-3}$ This tumor occurs possibly subsequent to trauma, for example, subcutaneous injections. ${ }^{1-4}$ The prevalence varies from 1:1,000 injections to $1: 10,000$ injections depending on the study. ${ }^{4}$ The post-injection inflammatory reactions lead to fibroblast proliferation and malignant transformation. As a preventive measure, recommendations as to injection sites have been formulated, notably the avoidance of injections in the interscapular region. ${ }^{4}$

Histopathologically, most FISSs are fibrosarcomas; however, other types of sarcomas can develop, including rhabdomyosarcoma, osteosarcoma, chondrosarcoma, malignant fibrous histiocytoma, or other high-grade stromal sarcomas. ${ }^{3}$ Following surgical treatment, this tumor may recur despite wide surgical excision. ${ }^{4,5}$ Other therapies such as radiotherapy and chemotherapy are proposed; nevertheless, and despite these treatments, medical requirements remain unmet. ${ }^{4}$ Recently, new immunotherapy treatment (Oncept IL-2 ${ }^{\circledR}$; Merial, Lyon, France) has been proposed to complement surgery and radiotherapy for feline fibrosarcomas without metastasis or lymph node lesions. This treatment consists of a local injection of recombinant poxviruses expressing interleukin-2, a previous clinical study having shown that the risk of recurrence is thus reduced. ${ }^{6}$ 
Masitinib is a tyrosine kinase inhibitor (TKI) approved for the treatment of canine mast cell tumors. ${ }^{7,8}$ In oncology, no clinical trials with masitinib have been published for feline patients. This compound has an interesting inhibitory profile to address FISS, selectively and potently inhibiting the tyrosine kinase receptors c-Kit, Lyn, and platelet-derived growth factor receptor (PDGFR). In the past, clinical case reports with imatinib (Gleevec ${ }^{\circledR}$ or Glivec ${ }^{\circledR}$; Novartis, Basel, Switzerland), another TKI, have demonstrated tumoral stabilization in the treatment of FISS. ${ }^{9}$ The observation set out below describes a therapeutic test with masitinib in a case of FISS. Obviously it cannot be considered predictive for other cases. The objective of this observation was to instigate further studies in order to assess the therapeutic relevance of TKIs in FISS.

\section{Case report}

In March 2012, an 8-year-old castrated and vaccinated male European shorthaired cat weighing $4 \mathrm{~kg}$ was presented for consultation with a $2 \mathrm{~cm}$ deep thoracodorsal subcutaneous tumor measuring $3 \times 6 \mathrm{~cm}$. It was painless, firm, and multinodular on palpation, adherent to deep tissues, and according to the owners had shown a rapid evolution within approximately 1-2 months. The most recent injections in this thoracodorsal area had been performed a year previously for vaccines against feline viral rhinotracheitis, calicivirus, panleukemia, feline leukemia virus, and rabies. Further examinations gave normal results: absence of satellite adenopathy, absence of pulmonary metastasis on X-ray examination, negative feline leukemia virus, and feline immunodeficiency virus blood tests and normal hematology and biochemistry values. Further to the owner's financial choice, computed tomography and magnetic resonance imaging were not performed. With the very strong suspicion of FISS and taking into account the rapid tumor progression, prompt wide surgical resection was the best option, with the objective of clarifying the diagnosis from the excisional tumor. To avoid delay, a prior biopsy was not deemed essential. A large surgical excision was made. Perioperative pain was monitored as usual using opiates and non-steroidal anti-inflammatories. The excision was unfortunately incomplete. A small portion of the tumor measuring $1 \times 3 \mathrm{~cm}, 0.5 \mathrm{~cm}$ deep, situated on the periphery, and remaining at the end of the surgery, was not removed to avoid widespread tissue damage and excessive tension on the stitches. This small tumor corresponded to a local extension of the primary tumor.

Histological analysis confirmed a lesion of feline fibrosarcoma complex (malignant fibrous histiocytoma), but no associated panniculitis lesion. The tumor was nonencapsulated and infiltrative. The prognosis was reserved because of the risk of potential local recurrence ( $75 \%$ of cases within 6-9 months) and development of metastases (25\% of cases). The median survival is estimated at $16-19$ months. ${ }^{4}$ Adjuvant radiotherapy and chemotherapy was fully indicated in this situation but was denied by the owners.

When the stitches were removed, the remaining tumor had already spread and increased by half. It was still painless and without edema, but firm and multinodular. No signs of postoperative inflammatory reaction were noted. Several options were again offered to the owner, including reexcision, chemotherapy, and radiotherapy. Immunotherapy with Oncept IL-2 was not suggested since, at the time, this treatment was not yet licensed. Targeted therapy of masitinib (Masivet 50 ${ }^{\circledR}$; AB Science, Paris, France) on an experimental basis was accepted and administered at a dose of $50 \mathrm{mg}$ orally on alternate days (off-label prescription). Masitinib was chosen on the basis of its profile as a TKI, and being a licensed medicine for veterinary use. This dosage was determined based on previous studies published on pharmacokinetics and toxicity in cats ${ }^{10,11}$ and on a clinical trial with a feline model of chronic allergic asthma. ${ }^{12}$ No anti-inflammatory medication was prescribed. Renal and liver functions were monitored according to the company guidance when using masitinib in dogs.

On re-evaluation after one month, the tumor was stable. After 2 months, the tumor had disappeared. The treatment was suspended by the owners in the third month due to mild side effects including nausea and vomiting, and their departure on holiday. Side effects were essentially observed in the first month of treatment and decreased gradually in intensity with time. It would have perhaps been appropriate to resume treatment.

In February 2013, 7 months after discontinuing masitinib, spherical tumoral recurrence with a diameter of $1.5 \mathrm{~cm}$ appeared beneath the scar. Treatment with masitinib was resumed at the same dosage, ie, $50 \mathrm{mg}$ orally every other day. No anti-inflammatory medication was associated. After 3 weeks, the tumor appeared stabilized and not adherent on palpation. The treatment being well tolerated by the patient, the dosage was doubled to $50 \mathrm{mg}$ per day $(12.5 \mathrm{mg} / \mathrm{kg} /$ day $)$, and this treatment was fairly well tolerated, with a slight loss of appetite. Administered over a further 5 weeks, the treatment resulted in slight tumoral regression (diameter of $1.3 \mathrm{~cm}$ ) measured over the widest diameter. The tumor being virtually stable, surgical excision was performed. Of note, the procedure was facilitated by the lack of adhesions 
to surrounding tissues. Histological analysis reconfirmed a lesion of feline fibrosarcoma complex (fibrosarcoma sensu stricto). The tumoral recurrence was nonencapsulated and locally infiltrative. No postoperative problems were observed.

As a preventive measure against recurrence, treatment with masitinib is currently ongoing for a period of several years at a dosage of $50 \mathrm{mg}$ on alternate days. Return to the initial dosage on alternate days is justified by the concern to minimize the possible long-term side effects, which are still fairly unknown in the cat. This treatment has been very well tolerated for over a year, with no side effects. No recurrence has been noted to date.

In summary, use of masitinib in this case of FISS had a positive therapeutic effect. The dosage used, ie, $12.5 \mathrm{mg} / \mathrm{kg}$ on alternate days and then daily, was well tolerated. The secondary effects on the digestive system were acceptable and reversible, and were mainly seen during the first month of treatment. The therapeutic response appeared within approximately one month. Therapeutic efficiency was less pronounced during recurrence, which is frequently more resistant. The duration of treatment favored in this pathology could be several years. Over the long term, the cost of treatment remains very reasonable.

\section{Discussion}

This therapeutic test is the first description of the use of masitinib in the treatment of FISS. Masitinib was prescribed off-label here, under the veterinarian's sole responsibility. For this reason, masitinib was not used as the first-line treatment. In the event of failure, the owners could have accused the veterinarian of having deprived his pet of other opportunities. The surgical option was thus chosen twice to halt tumor development. When recurrence occurred, continuation of treatment with masitinib was not justified once tumor stabilization had been confirmed. Moreover, surgical excision of the regrowth enabled observation of a decrease in adhesions further to preoperative treatment with masitinib. Such preoperative treatment facilitating surgery could become a relevant indication of masitinib.

Current publications recommend very extensive surgical excision to reduce the risk of recurrences. ${ }^{4,5,13}$ During the first operation, a small tumor corresponding to a local extension of the primary tumor could not be removed. Undoubtedly, this small tumor could have been removed by enlarging the surgical wound, but this would have caused additional pain and surgical complications.

During the initial treatment with masitinib, the tumor recurred within 7 months following the discontinuing of medication, corresponding to the average time scale for recurrence. The theory can be advanced that the effect of masitinib could cease on discontinuing treatment. For this reason, masitinib is currently prescribed as a preventive measure on a very long-term and possibly lifelong basis.

The therapeutic use of masitinib in FISS is based on scientific evidence. Masitinib is a TKI inhibiting in particular the following receptors: c-Kit, Lyn, and PDGFR, in which the PDGF ligand is an important cellular growth factor, especially for fibroblasts. ${ }^{14}$ Among the TKIs authorized for the veterinary market, masitinib is one of the most selective ${ }^{15}$ and well tolerated in cats. ${ }^{11}$

Recently, the antiproliferative and proapoptotic activity of masitinib was demonstrated in FISS cells in vitro. ${ }^{16,17}$ This action would mainly be the result of inhibition of the PDGFR, which is hyperactivated in FISS. Blockade of this growth factor receptor signaling pathway would thus inhibit cellular growth. ${ }^{16,17}$

Masitinib may function by an additional mode of action. Recently, mast cells have been implicated for their role in the development of tumors and metastasis, by increasing inflammation and immunosuppression in the tumoral microenvironment. ${ }^{18}$ By specifically inhibiting the activity of mast cell, masitinib would thus have an indirect antitumoral effect. ${ }^{18}$

Imatinib, the main TKI in human medicine and also a PDGFR inhibitor, has been tested in the past in the treatment of FISS. The results were favorable both in vitro and in vivo. ${ }^{9,19}$ Stabilization or moderate regression of tumors was noted in the treatment of four cats over an average duration of 2 months. ${ }^{9}$ However, the very high cost of the treatment thwarted any further studies and its use in veterinary medicine. On the other hand, masitinib is already licensed for veterinary use and compares favorably regarding the daily cost of treatment.

The case report described here involves only one therapeutic test. In the future, further studies on a large number of feline patients will enable a precise assessment of the therapeutic results of masitinib. The response to treatment could depend on the types of cells involved. More extensive and more detailed studies could be undertaken, especially in view of determining the responsive cellular types and/or associated markers. Study of the single clinical case above is not sufficient to be able to draw a conclusion on the higher sensitivity of malignant fibrous histiocytoma in comparison with fibrosarcoma sensu stricto.

The therapeutic potential of masitinib may be present at several levels. First, it may be effective in initial or preoperative treatment to reduce the size of the tumor or to facilitate 
excision by reducing adhesions. Second, it may be useful in prevention or treatment of recurrences and metastases. Third, it may have a role as an additional treatment, particularly in cases of inoperable FISS in combination with other therapies, such as radiotherapy, chemotherapy, and immunotherapy with Oncept IL-2. Should the tumor not disappear, both the control of its progression and tumoral stabilization by means of an inexpensive and fairly well tolerated treatment are of true therapeutic relevance.

In comparison, in human medicine, several TKIs (including imatinib and pazopanib [Votrient ${ }^{\circledR}$; GlaxoSmithKline, Brentford, UK]) are currently being tested with some success in the treatment of dermatofibrosarcomas and fibrosarcomas, both of which are pathologies where PDGFR is also abnormally hyperactivated. ${ }^{20-23}$ Regarding imatinib, a partial or fully favorable response can be noted in $50 \%$ of cases. ${ }^{22,23}$ Another indication for TKIs in human medicine is preoperative treatment during 2 or 3 months as a means of facilitating surgical excision by reduction of adhesions, ${ }^{21-23}$ also indicated in this case report. The new TKIs now under development will certainly improve on these results; a recent example has been published on a case of dermatofibrosarcoma resistant to radiotherapy and imatinib but sensitive to another recent TKI (sorafenib). ${ }^{24}$

In conclusion, a study of the use of TKIs, especially masitinib, in the treatment and prevention of recurrences of FISS would be interesting. Preoperative use to improve the surgical excision of tumors by reduction of adhesions and stabilization of inoperable FISS deserves to be studied specifically.

\section{Disclosure}

$\mathrm{PB}$ is employed by $\mathrm{AB}$ Science. $\mathrm{TC}$ was an employee of $\mathrm{AB}$ Science until December 2012. No other potential conflicts of interest are disclosed. The authors received no grant from any funding agency in the public, commercial, or not-for-profit sectors in relation to this work.

\section{References}

1. Kass PH, Spangler WL, Hendrick MJ, et al. Multicenter case-control study of risk factors associated with development of vaccine-associated sarcomas in cats. J Am Vet Med Assoc. 2003;223(9):1283-1292.

2. Srivastav A, Kass PH, McGill LD, Farver TB, Kent MS. Comparative vaccine-specific and other injectable-specific risks of injection site sarcomas in cats. J Am Vet Med Assoc. 2012;241(5):595-602.

3. Wilcock B, Wilcock A, Bottoms K. Feline postvaccinal sarcoma: 20 years later. Can Vet J. 2012;53:430-434.

4. Ladlow J. Injection site-associated sarcoma in the cat: treatment recommendations and results to date. J Feline Med Surg. 2013;15(5): 409-418.
5. Phelps HA, Kuntz CA, Milner RJ, Powers BE, Bacon NJ. Radical excision with five-centimeter margins for treatment of feline injection site sarcomas: 91 cases (1998-2002). J Am Vet Med Assoc. 2011;239(1): 97-106.

6. Jourdier TM, Moste C, Bonnet MC, et al. Local immunotherapy of spontaneous feline fibrosarcomas using recombinant poxviruses expressing interleukin 2 (IL2). Gene Ther. 2003;10(26):2126-2132.

7. Hahn KA, Ogilvie G, Rusk T, et al. Masitinib is safe and effective for the treatment of canine mast cell tumors. JVet Intern Med. 2008;22(6): 1301-1309.

8. Smrkovski OA, Essick L, Rohrbach BW, Legendre AM. Masitinib mesylate for metastatic and non-resectable canine cutaneous mast cell tumours. Vet Comp Oncol. July 12, 2013. [Epub ahead of print.]

9. Lachowicz JL, Post GS, Brodsky E. A phase I clinical trial evaluating imatinib mesylate (Gleevec) in tumor-bearing cats. J Vet Intern Med. 2005;19(6):860-864.

10. Bellamy F, Bader T, Moussy A, Hermine O. Pharmacokinetics of masitinib in cats. Vet Res Commun. 2009;33(8):831-837.

11. Daly M, Sheppard S, Cohen N, et al. Safety of masitinib mesylate in healthy cats. J Vet Intern Med. 2011;25(2):297-302.

12. Lee-Fowler TM, Guntur V, Dodam J, Cohn LA, DeClue AE, Reinero CR. The tyrosine kinase inhibitor masitinib blunts airway inflammation and improves associated lung mechanics in a feline model of chronic allergic asthma. Int Arch Allergy Immunol. 2012;158(4):369-374.

13. Martano M, Morello E, Buracco P. Feline injection site sarcoma: past, present and future perspectives. Vet J. 2011;188:136-141.

14. Dubreuil P, Letard S, Ciufolini M, et al. Masitinib (AB1010), a potent and selective tyrosine kinase inhibitor targeting KIT. PLoS One. 2009;4(9):e7258.

15. Anastassiadis T, Deacon SW, Devarajan K, Ma H, Peterson JR. Comprehensive assay of kinase catalytic activity reveals features of kinase inhibitor selectivity. Nat Biotechnol. 2011;29(11):1039-1045.

16. Lawrence J, Saba C, Gogal R Jr, et al. Masitinib demonstrates antiproliferative and pro-apoptotic activity in primary and metastatic feline injection site sarcoma cells. Vet Comp Oncol. 2012;10(2):143-154.

17. Feldhaeusser BR, Turek M, Lawrence J, Cornell K, Gogal RM Jr. Influence of different cell storage/culture conditions on spontaneous proliferation and level of tyrosine kinase receptor inhibition in two feline injection site sarcoma cell lines. J Immunoassay Immunochem. 2013;34(3):266-282.

18. Huang B, Lei Z, Zhang G-M, et al. SCF-mediated mast cell infiltration and activation exacerbate the inflammation and immunosuppression in tumor microenvironment. Blood. 2008;112(4):1269-1279.

19. Katayama R, Huelsmeyer MK, Marr AK, Kurzman ID, Thamm DH, Vail DM. Imatinib mesylate inhibits platelet-derived growth factor activity and increases chemosensitivity in feline vaccine-associated sarcoma. Cancer Chemother Pharmacol. 2004;54(1):25-33.

20. Labropoulos SV, Fletcher JA, Oliveira AM, Papadopoulos S, Razis ED. Sustained complete remission of metastatic dermatofibrosarcoma protuberans with imatinib mesylate. Anticancer Drugs. 2005;16(4): 461-466.

21. Kérob D, Porcher R, Vérola $\mathrm{O}$, et al. Imatinib mesylate as a preoperative therapy in dermatofibrosarcoma: results of a multicenter phase II study on 25 patients. Clin Cancer Res. 2010;16(12):3288-3295.

22. Malhotra B, Schuetze SM. Dermatofibrosarcoma protuberans treatment with platelet-derived growth factor receptor inhibitor: a review of clinical trial results. Curr Opin Oncol. 2012;24(4):419-424.

23. Ugurel S, Mentzel T, Utikal J, et al. Neoadjuvant imatinib in advanced primary or locally recurrent dermatofibrosarcoma protuberans: a multicenter phase II DeCOG trial with long-term follow-up. Clin Cancer Res. 2014;20(2):499-510.

24. Kamar FG, Kairouz VF, Sabri AN. Dermatofibrosarcoma protuberans (DFSP) successfully treated with sorafenib: case report. Clin Sarcoma Res. 2013;3(1):5. 
Veterinary Medicine: Research and Reports

Dovepress

\section{Publish your work in this journal}

Veterinary Medicine: Research and Reports is an international, Visit http://www.dovepress.com/testimonials.php to read real quotes peer-reviewed, open access journal publishing original research,

case reports, editorials, reviews and commentaries on all areas of

veterinary medicine. The manuscript management system is completely online and includes a very quick and fair peer-review system.

from published authors.

Submit your manuscript here: http://www.dovepress.com/veterinary-medicine-research-and-reports-journal 